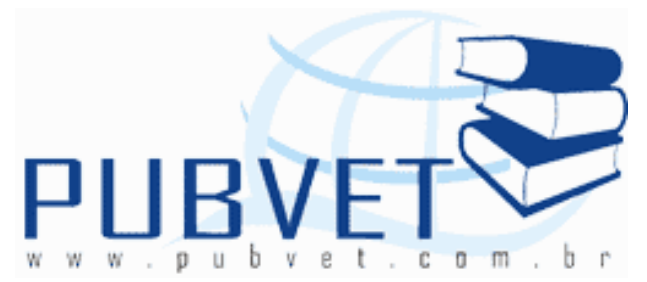

PUBVET, Publicações em Medicina Veterinária e Zootecnia.

\title{
Anestesia de tartaruga-da-amazônia Podocnemis expansa (Schweigger, 1812)-Testudines, Podocnemididae, com a associação cetamina e propofol
}

André Luiz Quagliatto Santos ${ }^{1}$, Lívia Monteiro Magalhães ${ }^{3}$, Cirilo Antônio de Paula Lima ${ }^{1}$, Liliane Rangel Nascimento ${ }^{2}$, Lorena Tannús Menezes ${ }^{2}$, Árthur Paulino Sanzo Kaminishi ${ }^{2}$, José Roberto Ferreira Alves Júnior ${ }^{4}$, Raul Henderson Avila Junior ${ }^{2}$

Laboratório de Ensino e Pesquisa em Animais Silvestres - LAPAS, FAMEV/UFU, e-mail: quagliatto@famev.ufu.br 1. Docente. 2. Mestrandos. 3. Médica Veterinária. 4. Doutorando.

\section{Resumo}

O objetivo desse estudo foi analisar os efeitos da associação dos anestésicos cetamina e propofol em Podocnemis expansa (tartaruga-da-amazônia). Utilizaram-se 20 cágados, que foram divididos em dois grupos de dez animais cada. O primeiro grupo recebeu cetamina $20 \mathrm{mg} / \mathrm{Kg} / \mathrm{IM}$ e propofol 10 $\mathrm{mg} / \mathrm{Kg} / \mathrm{IV}$ e o segundo grupo recebeu cetamina $60 \mathrm{mg} / \mathrm{Kg} / \mathrm{IM}$ e propofol 10 $\mathrm{mg} / \mathrm{Kg} / \mathrm{IV}$. Os animais foram pesados e identificados. A freqüência cardíaca, de cada animal, foi monitorizada antes de executar a anestesia e aos 10, 30, 60, 120 e 180 minutos após a administração dos anestésicos. As aplicações dos anestésicos ocorreram no membro torácico esquerdo (cetamina) e no seio vertebral cervical (propofol). Foram avaliados os graus de locomoção, 
SANTOS, A.L.Q. et al. Anestesia de tartaruga-da-amazônia Podocnemis expansa (Schweigger, 1812)-Testudines, Podocnemididae, com a associação cetamina e propofol. PUBVET, Londrina, V. 5, N. 19, Ed. 166, Art. 1120, 2011.

relaxamento muscular, facilidade de manipulação e resposta ao estímulo doloroso nos membros torácicos e pelvinos, após a administração dos anestésicos. Todos os animais entraram em plano anestésico cirúrgico antes de um minuto depois da aplicação dos anestésicos e mantiveram-se nesta condição, pelos 180 minutos em que os cágados foram avaliados.

Palavras-chave: anestesia, Podocnemis expansa, réptil, cetamina, propofol.

\title{
Anesthesia of the giant amazon river turtle Podocnemis expansa (Schweigger, 1812 -Testudines, Podocnemididae), with combination of ketamine and propofol
}

\begin{abstract}
The aim of this study was to analyze the effects of the combination of the ketamine and the propofol anesthetics in Podocnemis expansa (giant amazon river turtle). Were used 20 turtle, which were divided into two groups of ten animals each. The first group received ketamine $20 \mathrm{mg} / \mathrm{kg} / \mathrm{IM}$ and propofol $10 \mathrm{mg} / \mathrm{kg} / \mathrm{IV}$ and the second group received ketamine $60 \mathrm{mg} / \mathrm{kg} / \mathrm{IM}$ and propofol $10 \mathrm{mg} / \mathrm{kg} / \mathrm{IV}$. The animals were weighed and identified. The cardiac frequency of each animal was monitored before running the anesthesia and at $10,30,60,120$ and 180 minutes after administration of anesthetics. The applications of anesthetics occurred in the left forelimb (ketamine) and in the cervical vertebral sinus (propofol). Was assessed the degree of movement, muscle relaxation, ease of handling and response to painful stimulus in the thoracic limbs and pelvic, after administration of anesthetics. All animals entered in the surgical anesthesia plan before one minute after application of the anesthetic and remained in this condition by 180 minutes during the turtle's evaluation.
\end{abstract}

Key words: anesthesia, Podocnemis expansa, reptile, ketamine, propofol. 
SANTOS, A.L.Q. et al. Anestesia de tartaruga-da-amazônia Podocnemis expansa (Schweigger, 1812)-Testudines, Podocnemididae, com a associação cetamina e propofol. PUBVET, Londrina, V. 5, N. 19, Ed. 166, Art. 1120, 2011.

\section{INTRODUÇÃO}

A espécie Podocnemis expansa, popularmente conhecida como tartaruga-da-amazônia, é largamente distribuída pela bacia amazônica, ocorrendo em quase todos os afluentes do rio Amazonas. Sua distribuição é ampla nos estados do Amapá, Pará, Amazonas, Rondônia, Acre, Roraima, Tocantins, Goiás e Mato Grosso, englobando ecossistemas da floresta equatorial e do cerrado, na região Centro Oeste (MUNDIM et al., 1999).

Segundo Alho et al. (1979), Rodrigues (1992) e Molina e Rocha (1996), a Podocnemis expansa é o maior quelônio de água doce da América do Sul, podendo chegar a medir 75 a $107 \mathrm{~cm}$ de comprimento por 50 a $75 \mathrm{~cm}$ de largura (RODRIGUES, 1992) e pesando cerca de $60 \mathrm{~kg}$ de peso vivo (SMITH, 1979). Atualmente é considerada uma das espécies silvestres mais exploradas zootecnicamente. A exoticidade, o sabor e a qualidade de sua carne alcançam altos valores de mercado quando comparada com as carnes dos animais domésticos tradicionais (DORNELLES; QUINTANILHA, 2003).

Muitos são os acidentes que podem ocorrer com esses quelônios, principalmente causados pela atividade de pesca predatória, sendo necessária, muitas vezes, a intervenção cirúrgica, para solucionar os problemas causados por tais acidentes. Daí a importância de se ter o conhecimento da ação de cada anestésico e sua influência sobre os diferentes órgãos e tecidos, bem como os efeitos colaterais causados por eles.

Bennett (1991) e Boyer (1992), ao estudarem anestesia em quelônios, observaram que muitas técnicas foram testadas, mas a maioria apresentava limitações e efeitos colaterais, demonstrando que conhecimentos de sedação e anestesia são limitados nesses animais.

Anestésicos dissociativos e benzodiazepínicos são alguns dos fármacos mais utilizados para a anestesia em répteis. Os dissociativos necessitam de doses elevadas para atingir anestesia e apresentam períodos de recuperação 
SANTOS, A.L.Q. et al. Anestesia de tartaruga-da-amazônia Podocnemis expansa (Schweigger, 1812)-Testudines, Podocnemididae, com a associação cetamina e propofol. PUBVET, Londrina, V. 5, N. 19, Ed. 166, Art. 1120, 2011.

prolongados. Já os benzodiazepínicos são utilizados principalmente em associações com os derivados da fenciclidina (SCHILLIGER, 2000).

A cetamina e a tiletamina, únicos representantes da classe das fenciclidinas, encontram-se atualmente em uso clínico. O amplo emprego destes agentes, em medicina veterinária, deve-se ao fato de possuírem elevada margem de segurança, quando comparados aos barbitúricos, por haver a possibilidade de administração por outras vias além da intravenosa e por serem utilizados em inúmeras espécies de animais domésticos e silvestres (SPINOSA; GÓRNIAK, 1999).

Segundo Branson (2003), com o uso da cetamina, aumenta-se a freqüência cardíaca, há ausência de relaxamento muscular, não se verifica perda de reflexos protetores, permanecendo os olhos abertos e pupilas midriáticas e quando administrada isoladamente provoca analgesia intensa no sistema muscular esquelético.

A cetamina é considerada um fármaco que aumenta a tonicidade simpática, com elevação significativa da freqüência cardíaca, débito cardíaco, pressão arterial sistêmica, trabalho do ventrículo esquerdo e consumo de oxigênio pelo miocárdio (LIN, 1996).

O propofol é um anestésico geral, lipossolúvel de rápida ação e depuração. Induz depressão no sistema nervoso central, por intensificar os efeitos inibitórios do neurotransmissor ácido gama-aminobutírico (GABA) e por reduzir a atividade metabólica cerebral (THURMON; TRANQUILLI, 1996).

O propofol diminui a pressão arterial e conseqüentemente o débito cardíaco, porém com mínima alteração na freqüência cardíaca (ALVES; DOREA; ANDRADE, 2002). Produz um período hábil anestésico em cães de 10 a 15 minutos, produzindo discreta hipotensão e taquicardia sem alterações dignas de nota (MASSONE, 1999).

Frye (1991) acredita que o uso do Doppler é o melhor caminho para a aferição da freqüência cardíaca, pois o aparelho produz sons audíveis.

Hubbel (2003) indica, como monitor de pressão sanguínea, sondas de fluxo de Doppler como confiável para detectar pulsos periféricos, colocando-a 
SANTOS, A.L.Q. et al. Anestesia de tartaruga-da-amazônia Podocnemis expansa (Schweigger, 1812)-Testudines, Podocnemididae, com a associação cetamina e propofol. PUBVET, Londrina, V. 5, N. 19, Ed. 166, Art. 1120, 2011.

sobre uma artéria periférica. Cria-se um sinal audível à medida que pulsos de sangue passam por baixo da sonda e as ondas sonoras emitidas pelo Doppler são alteradas.

Nos zoológicos brasileiros, veterinários ao invés de utilizarem o eletrocardiograma, recomendam o Doppler vascular para o monitoramento da freqüência cardíaca de quelônios, devido a sua facilidade de uso, além de também apresentar parâmetros seguros para trabalhar a anestesia. Para tanto, faz-se necessário avaliar os valores normais da espécie para que o Doppler possa ser utilizado com segurança no diagnóstico de doenças ou para monitorar a anestesia (CARVALHO, 2005).

O objetivo do trabalho foi analisar os efeitos da associação dos anestésicos cetamina e propofol em Podocmenis expansa, após serem submetidas a dois protocolos anestésicos: cetamina $20 \mathrm{mg} / \mathrm{Kg} / \mathrm{IM}$ e propofol $10 \mathrm{mg} / \mathrm{kg} / \mathrm{IV}$ e cetamina $60 \mathrm{mg} / \mathrm{Kg} / \mathrm{IM}$ e propofol $10 \mathrm{mg} / \mathrm{Kg} / \mathrm{IV}$, visando a definição de padrões de efeitos colaterais, sucessos e outros efeitos da associação destes anestésicos, em quelônios.

\section{MATERIAL E MÉTODOS}

Foram utilizadas 20 Podocnemis expansa (tartaruga-da-amazônia), do criatório comercial Fazenda Moenda da Serra, clinicamente saudáveis, com idade aproximada de três anos, de ambos os sexos e peso variando de 1,0 à 1,5 quilos. O experimento foi conduzido no município de Araguapaz, estado de Goiás, na região do rio Araguaia, local onde a espécie estudada é natural.

A pesquisa foi realizada no mês de fevereiro de 2006, onde as condições climáticas eram de tempo quente e úmido, sendo que a temperatura foi aferida pelo termômetro de máxima e mínima, e a umidade do ar, expressa em pressão parcial de vapor pelo psicrômetro.

Os animais foram capturados de seus tanques de engorda, com auxílio de tarrafas e posteriormente pesados e identificados no casco com numeração seqüencial. 
SANTOS, A.L.Q. et al. Anestesia de tartaruga-da-amazônia Podocnemis expansa (Schweigger, 1812)-Testudines, Podocnemididae, com a associação cetamina e propofol. PUBVET, Londrina, V. 5, N. 19, Ed. 166, Art. 1120, 2011.

Depois de selecionados, foram conduzidos ao local do estudo, onde permaneceram até o término do experimento. Antes da aplicação dos anestésicos os animais eram rapidamente avaliados quanto a sua resposta a estímulos (Figura 1).

A administração dos anestésicos cetamina e propofol ocorreu no membro torácico esquerdo e no seio vertebral cervical, respectivamente, mediante prévia anti-sepsia e uso de seringas esterilizadas de $3 \mathrm{~mL}$, acopladas a agulhas hipodérmicas $13 \times 0,45 \mathrm{~mm}$. Os espécimes foram divididos em dois grupos e anestesiados por diferentes protocolos: 0 grupo 1 (G1) recebeu seqüencialmente, em seringas separadas, cetamina $20 \mathrm{mg} / \mathrm{Kg} / \mathrm{IM}$ e propofol $10 \mathrm{mg} / \mathrm{Kg} / \mathrm{IV}$ e o grupo 2 (G2) cetamina $60 \mathrm{mg} / \mathrm{Kg} / \mathrm{IM}$ e propofol $10 \mathrm{mg} / \mathrm{Kg} / \mathrm{IV}$. Em ambos os grupos, obedeceu-se o intervalo de 15 minutos entre as aplicações dos dois anestésicos.

Os parâmetros anestésicos foram observados nos determinados tempos: $0,5,10,20,30,45,60,90,120,150$ e 180 minutos, sendo que tempo zero foi considerado o momento da administração das drogas.

Os parâmetros avaliados foram os seguintes:

1. Locomoção: capacidade de se locomover normalmente, dificuldade de locomoção e ausência de locomoção;

2. Relaxamento muscular: capacidade de manter a cabeça elevada, facilidade de estender os membros do animal;

3. Manipulação: facilidade de manipular manualmente (flexão e extensão) a cabeça, os membros, a cauda e a boca do animal;

4. Sensibilidade dolorosa dos membros torácicos e pelvinos: pinçamento das falanges com pinça hemostática Kelly reta de $16 \mathrm{~cm}$, na primeira trava;

5. Batimentos cardíacos: aferição dos batimentos cardíacos pelo Doppler vascular, nos tempos $0,10,30,60,120$ e 180 minutos.

Um escore subjetivo de 1 para efeito mínimo, 2 para efeito médio e 3 para máximo efeito foi utilizado para os três primeiros parâmetros. Para os 
SANTOS, A.L.Q. et al. Anestesia de tartaruga-da-amazônia Podocnemis expansa (Schweigger, 1812)-Testudines, Podocnemididae, com a associação cetamina e propofol. PUBVET, Londrina, V. 5, N. 19, Ed. 166, Art. 1120, 2011.

testes de sensibilidade dolorosa, a resposta ao pinçamento foi considerada escore 0 e, a ausência da resposta ao pinçamento foi considerada escore 1 .

Quadro 1: Critérios atribuídos para classificar um parâmetro anestésico em graus de anestesia

\begin{tabular}{|c|c|c|c|}
\hline & \multicolumn{3}{|c|}{ Graus de anestesia (escores) } \\
\hline $\begin{array}{l}\text { Parâmetro } \\
\text { avaliado }\end{array}$ & 1 & 2 & 3 \\
\hline Locomoção & $\begin{array}{l}\text { Locomove-se com } \\
\text { rapidez (fuga). }\end{array}$ & $\begin{array}{l}\text { Dificuldade de } \\
\text { locomoção. }\end{array}$ & $\begin{array}{l}\text { Ausência de } \\
\text { locomoção. }\end{array}$ \\
\hline $\begin{array}{c}\text { Relaxamento } \\
\text { muscular }\end{array}$ & $\begin{array}{l}\text { Grande resistência } \\
\text { à traçãao manual, } \\
\text { retração da cabeça. }\end{array}$ & $\begin{array}{c}\text { Média resistência à } \\
\text { tração manual, } \\
\text { cabeça não muito } \\
\text { firme. }\end{array}$ & $\begin{array}{c}\text { Sem resistência à } \\
\text { tração manual, } \\
\text { não firma a } \\
\text { cabeça. }\end{array}$ \\
\hline Manipulação & $\begin{array}{l}\text { Difícil manipulação } \\
\text { e abertura da boca. }\end{array}$ & $\begin{array}{l}\text { Média facilidade de } \\
\text { manipulação. }\end{array}$ & $\begin{array}{l}\text { Fácil manuseio e } \\
\text { abertura da boca. }\end{array}$ \\
\hline & 0 & 1 & \\
\hline $\begin{array}{l}\text { Estímulo } \\
\text { membro } \\
\text { torácico }\end{array}$ & Manifesta dor. & Não manifesta dor. & \\
\hline $\begin{array}{l}\text { Estímulo } \\
\text { membro } \\
\text { pelvino }\end{array}$ & Manifesta dor. & Não manifesta dor. & \\
\hline
\end{tabular}

\section{RESULTADOS}

Após 15 minutos da aplicação da cetamina, todos os animais apresentaram escore 2 para locomoção (andavam, porém sem a mesma habilidade que apresentavam sem o anestésico), escore 1 para relaxamento muscular (cabeça firme, membros recolhidos), escore 1 para manipulação (difícil manuseio do animal, incluindo abertura da boca), escore 0 para sensibilidade dolorosa dos membros torácicos e pelvinos (manifestavam dor quando estimulados) e elevação dos batimentos cardíacos. Além disso, apresentaram reflexo protetor óculo-palpebral.

Todos os répteis, que receberam o propofol, em seguida (quinze minutos após cetamina), obtiveram escore 11 , ou seja, escore 3 (máximo efeito) para 
SANTOS, A.L.Q. et al. Anestesia de tartaruga-da-amazônia Podocnemis expansa (Schweigger, 1812)-Testudines, Podocnemididae, com a associação cetamina e propofol. PUBVET, Londrina, V. 5, N. 19, Ed. 166, Art. 1120, 2011.

os parâmetros locomoção, 3 para relaxamento muscular e 3 para manipulação e escore 1 para sensibilidade dolorosa dos membros torácicos e 1 para membros pelvinos. Todos os animais entraram em plano anestésico cirúrgico, em menos de um minuto após a administração do anestésico (Figura 2).

Durante os 180 minutos em que os cágados foram avaliados, mantiveram escore 11 e os batimentos cardíacos diminuíram sua freqüência até próximo aos valores iniciais.

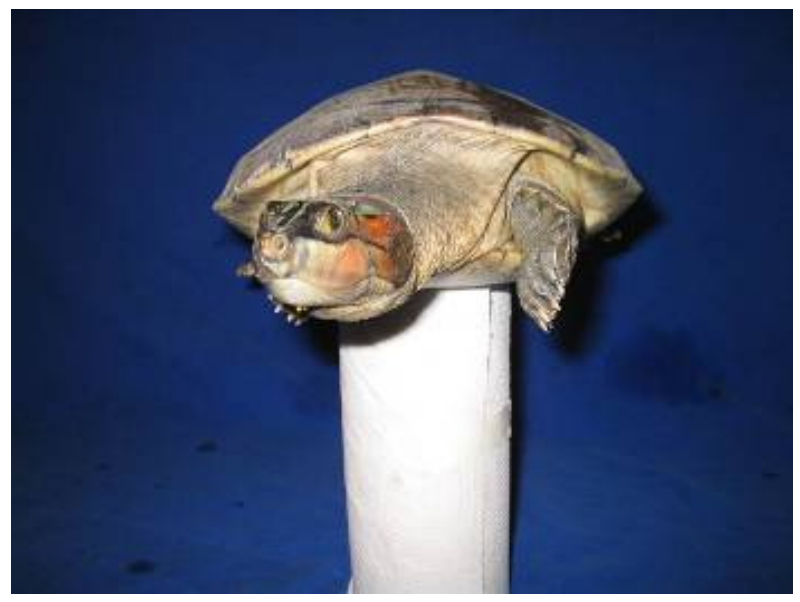

Figura 1: Fotografia de Podocnemis expansa, antes da aplicação dos anestésicos.

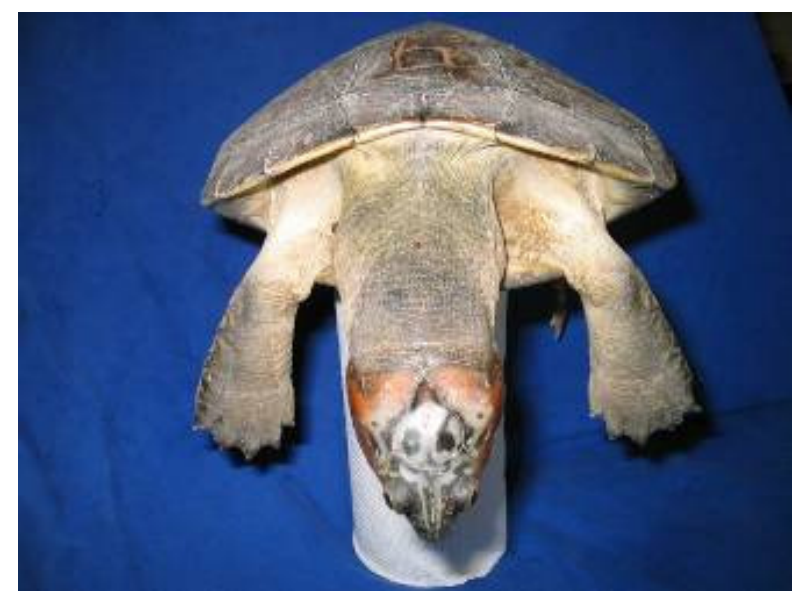

Figura 2: Fotografia de Podocnemis expansa, após a aplicação dos anestésicos 
SANTOS, A.L.Q. et al. Anestesia de tartaruga-da-amazônia Podocnemis expansa (Schweigger, 1812)-Testudines, Podocnemididae, com a associação cetamina e propofol. PUBVET, Londrina, V. 5, N. 19, Ed. 166, Art. 1120, 2011.

\section{DISCUSSÃO}

O estudo foi realizado em ambiente natural dos animais, onde a temperatura mínima foi de $23^{\circ} \mathrm{C}$, a máxima de $31^{\circ} \mathrm{C}$ e a umidade do ar, expressa em pressão parcial de vapor, foi de 3,56 KPa. Essa temperatura é considerada ótima, pois segundo Bennett (1991), é nesta condição que o animal apresenta seu metabolismo em melhor funcionamento. Portanto, foi esperada a maior ação possível das drogas utilizadas.

Os resultados obtidos, utilizando cetamina $20 \mathrm{mg} / \mathrm{Kg} / \mathrm{IM}$ e $60 \mathrm{mg} / \mathrm{Kg} / \mathrm{IM}$, estão de acordo com Branson (2003), quando afirma que, com o uso da cetamina, aumenta-se a freqüência cardíaca, há ausência de relaxamento muscular, não se verifica perda de reflexos protetores, permanecendo os olhos abertos.

Segundo Massone (1999), a cetamina, por apresentar uma ação simpatomimética, estimula a freqüência cardíaca, fato observado em Podocnemis expansa.

De acordo com Sebbel e Lowdon (1989), a injeção de um bolo de propofol resulta em alto índice de apnéia durante a indução. Pye e Carpenter (1998) afirmam que o propofol tem como efeito colateral hipotensão e apnéia. Os répteis, aqui estudados, manifestaram apnéia e não receberam ventilação assistida apresentando cianose da mucosa oral.

A ausência do músculo diafragma, nos répteis, implica em uma maior participação de outros músculos estriados durante a respiração, sendo que nos quelônios ocorre a participação de cinturões musculares torácicos e pelvinos (SCHILLIGER, 2000). Em planos anestésicos profundos pode ocorrer a paralisação desses músculos e uma conseqüente parada respiratória (BENNET, 1991). Tais afirmações podem explicar o fato da ocorrência da apnéia, presenciada nos animais ora anestesiados.

Pye e Carpenter (1998) utilizaram a associação de cetamina e propofol para a retirada de um corpo estranho do esôfago de uma Trachemys scripta. Relataram que o animal recebeu cetamina $30 \mathrm{mg} / \mathrm{Kg} / \mathrm{IM}$ e após 30 minutos o 
SANTOS, A.L.Q. et al. Anestesia de tartaruga-da-amazônia Podocnemis expansa (Schweigger, 1812)-Testudines, Podocnemididae, com a associação cetamina e propofol. PUBVET, Londrina, V. 5, N. 19, Ed. 166, Art. 1120, 2011.

animal estava relaxado o suficiente para isolar a veia jugular. Depois de 90 minutos, injetou-se propofol $7 \mathrm{mg} / \mathrm{Kg} / \mathrm{IV}$ no cágado e o plano anestésico cirúrgico foi alcançado em 30 segundos, com duração de aproximadamente 15 minutos. Este protocolo foi repetido com uma dosagem inferior de cetamina (25mg/Kg/IM), mas com a mesma de propofol, por mais duas vezes para exames pós-operatórios, com resultados similares e sem efeitos adversos. No entanto, a associação dos anestésicos cetamina e propofol, em Podocnemis expansa teve seu efeito prolongado.

\section{CONCLUSÃO}

Do exposto, conclui-se que os protocolos anestésicos, utilizados no experimento, foram eficazes para anestesia cirúrgica de Podocnemis expansa. No entanto, períodos prolongados de apnéia observados, podem resultar em risco de morte para os animais.

\section{REFERÊNCIAS}

ALHO, C. J. R.; CARVALHO, A. G.; PÁDUA, L. F. M. Ecologia da tartaruga da Amazônia e avaliação de seu manejo na Reserva Biológica do Trombetas. Brasil Florestal, Brasília, DF, v.9, n. 38, p. 29-47, 1979.

ALVES, T. C.; DOREA, E. M. L.; ANDRADE, R.F. Anestésicos gerais Intravenosos. In: SILVA, P. Farmacologia. 6 ed. Rio de Janeiro: Guanabara Koogan , 2002. p. 391-403.

BENNETT, R. A. A review of anesthesia and chemical restrain in reptiles. Journal of Zoo and Wild life Medicine, Philadelphia, v.22, p.282-303, 1991.

BOYER, T. H. Clinical anesthesia for Reptiles. Bulletin Association of Reptiles Amphibians Veterinarians, Paris, v.2, p. 10-13, 1992.

BRANSON, K. R. Anestésicos injetáveis. In: ADAMS, H. R. Farmacologia e terapêutica em veterinária. 8. ed. Rio de Janeiro: Guanabara Koogan, 2003, cap.12, p.208.

CARVALHO, S. F. M. Anestesia dissociativa em tartarugas da amazônia (Podocnemis expansa, Schweigger-1812) (Testudinata) e monitoramento cardíaco com eletrocardiógrafo. 2005. Dissertação (Mestrado) - Faculdade de Medicina Veterinária, Universidade Federal de Uberlândia, Uberlândia, 2005.

DORNELLES,A.M.G.; QUINTANILHA,L.C. Relatório do abate experimental da tartaruga-daamazônia (Podocnemis expansa) criada em cativeiro. Goiânia: IBAMA - RAN, 2003. 
FRYE, F. L. Anesthesia. In: FRYE, F. L. Reptile care na atlas of diseases and tratament. Neptune City: T. F. H. Publication, 1991. v. 2, p. 421-437.

HUBBELL, J. A. E. Métodos práticos de anestesia in manejo do paciente. In: BICHARD, S. J.; SHERDING, R. G. Manual saunders: clínica de pequenos animais, Tradução de Paulo Marcos Angria de Oliveira. 2. ed. São Paulo : Roca, 2003. Seção 1, cap. 2, p.14.

LIN, H. C. Dissociative anesthetics. In: LUMB, W. V.; JONES, E. W. Veterinary anaesthesia. 3. ed. Baltimore: Williams e Wilkins, 1996. cap. 10, p.241-296.

MASSONE, F. Medicação pré anestésica. Miorrelaxantes. In: MASSONE, F. Anestesiologia veterinária. 2. ed. Rio de Janeiro: Guanabara Koogan, 1999. cap.9, p.26.

MOLINA, F. B.; ROCHA, M. B. Identificação, caracterização e distribuição dos quelônios da Amazônia Brasileira. Belém: Centro Nacional dos Quelônios da Amazônia, 1996. 24p. Apostila.

MUNDIM, A. V.; QUEIROZ, R. P.; SANTOS, A. L. Q.; BELLETI, M. E.; LUZ, V. L. F. Bioquímica sanguínea da tartaruga da Amazônia (Podocnemis expansa) em seu habitat natural.

Bioscience Journal, Uberlândia, v. 15, n. 2, dez. 1999.

PYE, G. W.; CARPENTER, J. W. Ketamine sedation followed by propofol anesthesia in a slider (Trachemys scripta) to facilitate removal of an esophageal foreing body. Journal of Zoo and Wildlife Medicine, Lawrence, v. 29 n.3, p. 352, 1998.

RODRIGUES, R. M. Quelônios. In: A fauna da Amazônia. Belém: CEJUP, 1992. p.209-214.

SCHILLIGER, L. Anesthésie des reptiles: Anesthésie: reanimation. In: ENCYCLOPÉDIE Vétérinaire. Paris: Editions Scientifiques et Médicales Elsevier, 2000. 6p.

SEBBEL, P. S.; LOWDON, J. D. Propofol: a new intravenous anesthetic. Anesthesiology, Philadelphia, v.71, n. 1, p. 260-277, 1989.

SMITH, N. J. H. Quelônios aquáticos da Amazônia: um recurso ameaçado. Acta Amazônica, Manaus, v.9, n.1, p.87-97, 1979.

SPINOSA, H.S., GÓRNIAK, S.L. Tranqüilizantes e relaxantes musculares de ação central. In: SPINOSA, H.S., GÓRNIAK, S.L., BERNARDI, M. M. Farmacologia aplicada à medicina veterinária. 2. ed. Rio de Janeiro: Guanabara Koogan,1999. cap.14, p. 118, 119, 122, 141146.

THURMON, J. C.; TRANQUILLI, W. J. Injectable Anesthetics. In: THURMON, J. C.; TRANQUILLI, W. J. Lumb e Jones Veterinary Anesthesia, Pensylvania. p. 210-240, 1996. 